\title{
Eriocitrin and Apigenin as New Carbonic Anhydrase VA Inhibitors from a Virtual Screening of Calabrian Natural Products
}

\author{
Authors \\ Maria Concetta Gidaro ${ }^{1}$, Francesca Alcaro ${ }^{1}$, Simone Carradori ${ }^{2}$, Giosuè Costa ${ }^{1}$, Daniela Vullo ${ }^{3}$, Claudiu T. Supuran ${ }^{3}$, \\ Stefano Alcaro ${ }^{1}$ \\ Affiliations \\ ${ }_{1}^{1}$ Dipartimento di Scienze della Salute, Università “Magna Graecia” di Catanzaro, Campus “S. Venuta”, Catanzaro, Italy \\ 2 Dipartimento di Chimica e Tecnologie del Farmaco, Sapienza University of Rome, Rome, Italy \\ ${ }^{3}$ Laboratorio di Chimica Bioinorganica, Polo Scientifico, Università degli Studi di Firenze, Sesto Fiorentino, Florence, Italy
}

\author{
Key words \\ - Citrus bergamia \\ - Rutaceae \\ - Allium cepa var. Tropea \\ - Liliaceae \\ - flavonoids \\ - docking \\ - structure-based virtual \\ screening \\ - human carbonic anhydrase \\ inhibition
}

\section{Abstract}

$\nabla$

In this work, we performed a structure-based virtual screening against five carbonic anhydrase isoforms using, as a ligand library, natural components of Citrus bergamia (Bergamot) and Allium cepa var. Tropea (red onion) sources, which are some typical Calabrian products. The most relevant Bergamot and red onion components, identified as potentially new hits by means of the computational work, were submitted to in vitro tests in order to confirm the ability to exert the predicted biological activity. Apigenin and eriocitrin were identified as new potent inhibitors of human carbonic anhydrase VA isozyme.

$\begin{array}{ll}\text { Abbreviations } \\ \text { AZA: } & \text { acetazolamide } \\ \text { CA: } & \text { carbonic anhydrase } \\ \text { EFE: } & \text { Embrace free energy } \\ \text { LGS: } & \text { ligand G-score } \\ \text { hCA: } & \text { human carbonic anhydrase } \\ \text { hCAI: } & \text { human carbonic anhydrase inhibitor } \\ \text { mCA: } & \text { murine carbonic anhydrase } \\ \text { NPs: } & \text { natural products } \\ \text { PDB: } & \text { Protein Data Bank } \\ \text { PDO: } & \text { protected denomination of origin } \\ \text { PGI: } & \text { protected geographical indication } \\ \text { SBVS: } & \text { structure-based virtual screening } \\ \text { VS: } & \text { virtual screening } \\ \text { XGS: } & \text { X-ray G-score } \\ \text { ZN: } & \text { zinc atom }\end{array}$

Supporting information available online at http://www.thieme-connect.de/products

\section{Introduction \\ $\nabla$}

Drug discovery is the result of an expensive and time-consuming process; about 800 million to 1 billion dollars for each new compound. In this context, to speed the drug-discovery process, the identification of novel bioactive compounds can be adequately supported by using computational techniques. The advantages of these approaches can be in terms of time and/or resource savings in the drug development flowchart.

Healing properties of natural products have been known since ancient times and nowadays represent an important resource for the development of new drugs [1]. Computational methods, like VS experiments, reduce the overall cost and time associated with the discovery of new drugs from nature, by identifying in silico the most promising candidates to focus the experimental efforts on [2].
Data modeling approaches are used in both lead identification and lead optimization, and the "rational drug design" concept is based on the principle that biological properties of active compounds are related to their structural features [3, 4]. Today, this method is particularly useful in the field of nutraceutics, and, in our research group, the VS approach was recently applied against the DNA G-quadruplex human telomeric repeated sequence, combining ligand- and structure-based approaches used for the identification of the psoralen moiety as a privileged scaffold for the development of novel binders [5], and to search for new hits from commercially natural compound databases [6].

Our project, named DATATIPICA, was focused on chemical components belonging to selected typical agricultural foods from Calabria (Italy), including Bergamot (Citrus bergamia Risso, Rutaceae) and Tropea red onion (Allium cepa L. var. Tropea, 


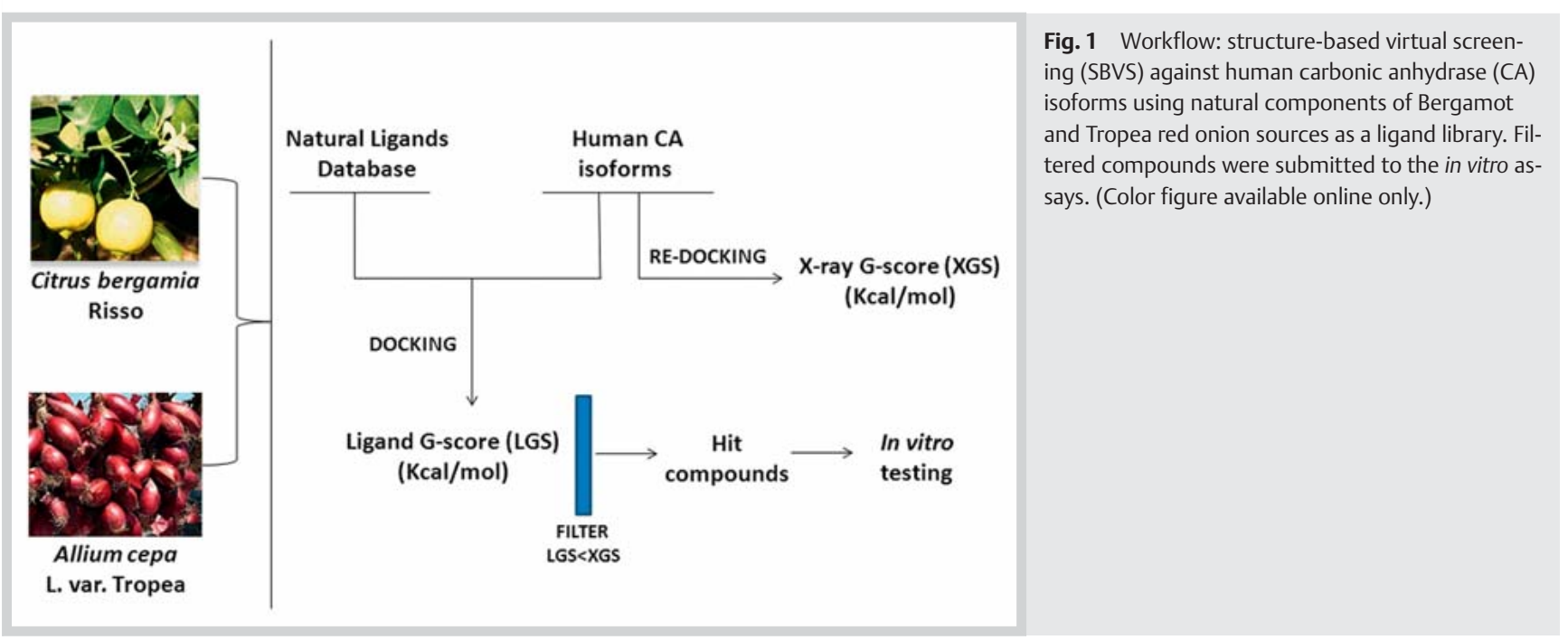

Liliaceae), with the purpose to discover new bioactive compounds as potential hCAIs.

In 2001, the Calabrian Bergamot source gained Italian designation PDO by the Bergamot Consortium of Reggio Calabria with a unique profile of free flavonoids and glycoside derivatives [7].

Moreover, Tropea red onion was recently certified as a PGI product from the European Union [8] and also represents a significant source of flavonoids [9], with a health benefit profile also shared by Bergamot, as confirmed by several pharmacological studies [10-14].

In the literature, there are many clinical trials regarding the biological effects of Bergamot and red onion, but there is no evidence of the possible mechanisms of action of its components. Conversely, our study was focused on a specific interaction between these contents and the CA family, a target involved in some pathophysiological processes.

hCAs are zinc-containing metalloenzymes that catalyze the reversible hydration of carbon dioxide according to the following reaction:

$\mathrm{CO}_{2}+\mathrm{H}_{2} \mathrm{O} \leftrightarrows \mathrm{HCO}_{3}^{-}+\mathrm{H}^{+}$

Sixteen hCA isozymes have been described up to now in mammals with variable kinetic parameters, expression levels, tissues/ organs, and subcellular localization [15-18]. Some of these isozymes (e.g., hCA II, VA, IX, XII) constitute targets for the development of antiglaucoma, diuretic, antiobesity, anticonvulsant, or anticancer drugs [19-22]. Supuran's group recently investigated the interactions of hCA I, II, VI, and bCA III isozymes with several types of flavonoids $[23,24]$ that may be used as leads for generating more potent hCAIs and, with this perspective, we carried out this study enlarging the possibility to discover new flavonoids as inhibitors of these metalloenzymes.

\section{Results}

$\nabla$

The SBVS was performed as a hierarchical procedure, combining different computational tools in order to reduce the false positive, sometimes predicted in silico (๑ Fig. 1).

Briefly, our procedure can be summarized in three steps: i) prediction, for each ligand, of binding poses using the Glide docking program; ii) energy minimization and rescoring of the best docked poses using the Embrace method; and iii) finally, selection of the best scored complexes to analyze ligand-target interactions.

All PDB [25] X-ray targets were downloaded with a co-crystallized inhibitor: AZA (11) in the hCA I, IX, and mCA VA X-ray structures (PDB codes: 1AZM, 1DMY, and 4HT2); 6-aminosaccharin (12) in the X-ray isoform of hCA II (PDB code: 4CQ0), and 4[(4,6-dimethylpyrymidin-2-yl)thio]-2,3,5,6-tetrafluorobenzenesulfonamide compound (13) in the X-ray isoform of hCA XII (PDB code: 4HT2), as shown in $\odot$ Fig. 2.

In order to perform a re-docking simulation, our protocol was applied to reproduce bioactive poses of the hCAIs (11-13) content in the crystallographic structures. XGS values of the best docked molecules, expressed in $\mathrm{Kcal} / \mathrm{mol}$, were used to select the new natural hCAIs from our library.

In fact, in a modeling study after the optimal binding poses have been predicted, the next step is to rank or score the generated structures to determine their relative binding affinities.

AZA (11) should be the common reference ligand for all targets, but, unfortunately, there are no hCA II and XII PDB models cocrystallized with it. Therefore, X-ray structures with 4CQ0 and 4HT2 PDB codes, respectively, co-crystallized with compounds 12 and 13, were used for the re-docking purpose ( $\bullet$ Table 1 ). Moreover, in order to consider the best-known inhibitor in our reference analysis, AZA complexes were generated with hCA isoforms II and XII also.

Analyzing the first step results reported in $\bigcirc$ Table 1, we concluded that ten of the 280 natural compounds from our database with LGS values, expressed in $\mathrm{Kcal} / \mathrm{mol}$, better than XGS for hCA I, XII, and mCA VA isoforms could act as hCAIs. The best ten compounds, 1-10, are reported in 0 Fig. 2. Respectively, compounds 1-5 are polyphenols prevalent in the Bergamot source, while 610 are from Tropea red onion. Interestingly, docking results of compounds $\mathbf{1 - 1 0}$ carried out with the hCA XII isozyme displayed some LGS performances better than the AZA (11) G-score value that is equal to $-5.47 \mathrm{Kcal} / \mathrm{mol}$ and not very dissimilar to the XGS $-5.80 \mathrm{Kcal} / \mathrm{mol}$ obtained with compound 13.

It has been noted that the use of multiple scoring functions in concert significantly improve hit rates compared to the use of a single scoring function [26]. For this reason, the targets with a better LGS of compounds 1-10 (hCA I, XII, and mCA VA) were sub- 
<smiles>[R]Oc1ccc(-c2cc(=O)c3c(O)cc(O)cc3oc2=O)cc1[R]</smiles>

Naringin (5)<smiles>[R]c1cc(-c2oc3cc(O)cc(O)c3c(=O)c2[R])ccc1O</smiles>

Quercetin (7)

Kaempferol $(9$

Isorhamnetin (10)<smiles>Nc1ccc2c(c1)S(=O)(=O)NC2=O</smiles>

6-aminosaccharin (12)<smiles>[R6]Oc1ccc([C@@H]2CC(=O)c3c(O)cc(O[C@@H]4O[C@H](CO[C@H]5OC(C)[C@H](O)[C@H](O)[C@H]5O)[C@@H](O)[C@H](O)[C@H]4O)cc3O2)cc1O</smiles>

Eriocitrin (3) $\mathrm{R}=\mathrm{H}$ Hesperidin (4) $\mathrm{R}=\mathrm{CH}_{3}$<smiles>O=C1c2c(O)cc(O)cc2O[C@@H](c2ccc(O)c(O)c2)[C@@H]1O</smiles>

Taxifolin (6)<smiles>CC(=O)Nc1nnc(S(N)(=O)=O)s1</smiles>

Acetazolamide (AZA) (11)<smiles>Cc1cc(C)nc(Sc2c(F)c(F)c(S(N)(=O)=O)c(F)c2F)n1</smiles>

4-[(4,6-dimethylpyrimidin-2-yl)thio]-2,3, 5,6 -tetrafluorobenzenesulfonamide

(13)
Fig. 2 Structures of: 1) the most relevant flavonoids (1-10) selected in an in silico study by a structure-based virtual screening method and submitted to in vitro assays for human carbonic anhydrase I, II, VA, IX, and XII isozymes; 2) acetazolamide (11), a co-crystallized inhibitor of human carbonic anhydrase I and IX, and murine carbonic anhydrase VA isozymes and a positive control for the in vitro assays; 3) 6-aminosaccharin (12), a human carbonic anhydrase II isozyme co-crystallized inhibitor; 4) 4[(4,6-dimethylpyrymidin-2-yl)thio]-2,3,5,6-tetrafluorobenzenesulfonamide (13), a human carbonic anhydrase XII isozyme co-crystallized inhibitor.

Table 1 G-score values in Kcal/mol: 1) ligand G-score of selected polyphenols (1-10) obtained by the best poses of docking simulations against human carbonic anhydrases I, II, IX, and XII, and murine carbonic anhydrase VA; 2) ligand G-score of acetazolamide (11) against human carbonic anhydrase II and XII isoforms; 3) * values are the X-ray G-score of reference compounds 11-13 computed by re-docking each target using, respectively, the Protein Data Bank models with entry codes 1AZM, 4CQ0, 1DMY, 3IAI, and 4HT2.

\begin{tabular}{|c|c|c|c|c|c|}
\hline \multirow[b]{2}{*}{ Compound } & \multicolumn{5}{|c|}{ G-score (Kcal/mol) } \\
\hline & hCA I & hCA II & mCA VA & hCA IX & hCA XII \\
\hline 1 & -5.85 & -5.53 & -6.39 & -6.02 & -5.50 \\
\hline 2 & -6.86 & -5.64 & -6.43 & -5.01 & -6.03 \\
\hline 3 & -6.50 & -5.18 & -6.40 & -5.55 & -6.27 \\
\hline 4 & -6.33 & -5.95 & -6.77 & -5.62 & -5.57 \\
\hline 5 & -5.93 & -4.99 & -5.05 & -5.38 & -5.73 \\
\hline 6 & -6.12 & -6.10 & -6.87 & -5.57 & -5.78 \\
\hline 7 & -6.09 & -5.82 & -6.71 & -5.61 & -5.16 \\
\hline 8 & -5.13 & -6.41 & -7.03 & -4.62 & -6.40 \\
\hline 9 & -5.68 & -5.94 & -6.70 & -5.90 & -5.40 \\
\hline 10 & -5.81 & -5.25 & -6.72 & -5.21 & -5.13 \\
\hline 11 & $-5.99^{*}$ & -6.70 & $-6.69^{*}$ & $-6.42^{*}$ & -5.47 \\
\hline 12 & 1 & $-7.40^{*}$ & 1 & 1 & 1 \\
\hline 13 & 1 & 1 & 1 & 1 & $-5.80^{*}$ \\
\hline
\end{tabular}




\begin{tabular}{|c|c|c|c|}
\hline \multirow[b]{2}{*}{ Compound } & \multicolumn{3}{|c|}{ EFE (Kcal/mol) } \\
\hline & hCA I & mCA VA & hCA XII \\
\hline 1 & -13.90 & -18.73 & -18.45 \\
\hline 2 & -7.48 & -21.61 & -15.04 \\
\hline 3 & -26.22 & -35.02 & -29.20 \\
\hline 4 & -16.54 & -22.28 & -28.87 \\
\hline 5 & -21.68 & -22.99 & -18.48 \\
\hline 6 & -16.96 & -18.77 & -13.32 \\
\hline 7 & -6.97 & -18.60 & -18.94 \\
\hline 8 & -3.70 & -20.82 & -15.26 \\
\hline 9 & -8.69 & -19.86 & -18.24 \\
\hline 10 & -8.80 & -18.36 & -2.11 \\
\hline 11 & -37.19 & -32.99 & -48.16 \\
\hline
\end{tabular}

Table 2 Embrace free energy (EFE in $\mathrm{Kcal} / \mathrm{mol}$ ) computed for compounds $\mathbf{1 - 1 1}$ against the targets human carbonic anhydrase I and XII, and murine carbonic anhydrase VA isozymes.

Table 3 Inhibition constant $K_{\mathrm{i}}$ values $(\mu \mathrm{M})$ of human carbonic anhydrase I, II, VA, IX, and XII isoforms for polyphenols 1-10 and acetazolamide (11).

\begin{tabular}{|c|c|c|c|c|c|}
\hline \multirow[b]{2}{*}{ Compound } & \multicolumn{5}{|c|}{ Inhibition constants $K_{\mathrm{i}}(\mu \mathrm{M})$} \\
\hline & hCA I & hCA II & hCA VA & hCA IX & hCA XII \\
\hline 1 & $4.10^{\mathrm{a}}$ & $2.70^{\mathrm{a}}$ & 0.30 & 0.46 & 1.00 \\
\hline 2 & 2.95 & 0.41 & 2.82 & 2.52 & 0.38 \\
\hline 3 & 3.13 & 0.31 & 0.15 & 3.57 & 2.18 \\
\hline 4 & $>10$ & 0.42 & 1.92 & 0.44 & 0.29 \\
\hline 5 & $>10$ & $>10$ & 2.10 & 3.39 & 0.24 \\
\hline 6 & 3.66 & 0.34 & 4.24 & 0.43 & 0.19 \\
\hline 7 & $3.60^{\mathrm{a}}$ & $2.40^{\mathrm{a}}$ & 2.65 & 2.22 & 0.45 \\
\hline 8 & $>10$ & 0.41 & 4.08 & 3.59 & 0.17 \\
\hline 9 & 2.53 & 0.26 & 3.93 & 0.45 & 0.11 \\
\hline 10 & $>10$ & 0.44 & $>10$ & 2.79 & 0.22 \\
\hline 11 & 0.10 & 0.008 & 0.38 & 0.041 & 0.038 \\
\hline
\end{tabular}

a Values from literature data [23]
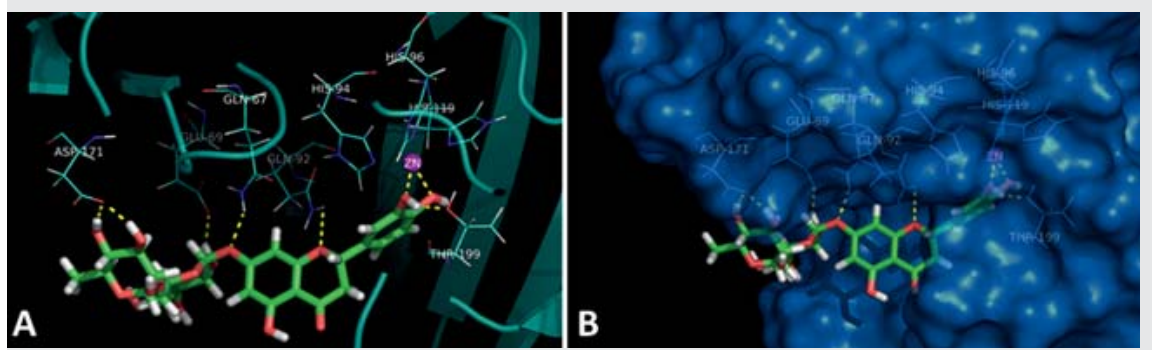

Fig. 3 Eriocitrin (3) best pose and its polar contacts/metal interaction into the murine carbonic anhydrase VA (PDB code 1DMY) binding cavity. A Eriocitrin (3) is rendered as carbon green sticks, the pocket amino acids are labeled as carbon cyan lines, the zinc atom as a purple sphere, and the target is described as a cyan cartoon. B Eriocitrin (3) is rendered as carbon green sticks, the pocket amino acids are labeled as blue lines, the zinc atom as a purple sphere, and the rest of the target is depicted as a blue surface. All non-carbon atoms are colored according to atom types. (Color figure available online only.)

mitted to a further computational step based on the complex free energy estimation, after a full energy minimization, by the Embrace method as implemented in the Schrödinger Software MacroModel [27].

This second step suggested a potential inhibition of eriocitrin (3) against the mCA VA isozyme. Actually, the result of its EFE, equal to $-35.02 \mathrm{Kcal} / \mathrm{mol}$, was better than the AZA (11) value of -32.99 $\mathrm{Kcal} / \mathrm{mol}(\odot$ Table 2).

Finally, polyphenols $\mathbf{1 - 1 0}$ were submitted to in vitro tests against all targets, using AZA (11) as a positive control in order to confirm the ability of our SBVS method to predict the biological activity against CA isoforms.

Eriocitrin (3) proved to be the best hCA VA inhibitor with a $K_{\mathrm{i}}$ value of $0.15 \mu \mathrm{M}$ against AZA (11), resulting in a $K_{\mathrm{i}}$ of $0.38 \mu \mathrm{M}$, as shown in Table 3. Conversely, the computational prediction failed for apigenin (1) with respect to the reference compound. In fact, despite its EFE value of $-18.73 \mathrm{Kcal} / \mathrm{mol}$ ( $\odot$ Table 2), it showed an inhibition performance slightly better than reference 11 ( Table 3).

With the aim to explain the possible mechanism of action of ten selected polyphenols, we analyzed in silico ligand-target interactions of the most active natural ligands $\mathbf{3}$ and $\mathbf{1}$ against mCA VA isozyme (PDB code 1DMY). As reported above, eriocitrin (3) fits into the binding site of mCA VA with an acceptable G-score value of $-6.40 \mathrm{Kcal} / \mathrm{mol}$ ( Table 1) and the best EFE value of -35.02 $\mathrm{Kcal} / \mathrm{mol}$ ( Table 2). The best docked pose, after Embrace minimization, is reported in $\odot$ Fig. 3 , where the molecule and its contact with amino acid residues of the target were visualized using Pymol version 1.7.0.0 [28]. 
The molecular modeling study suggests that the best pose of apigenin (1) is also located in the binding pocket of the MCA VA isoform, with an acceptable G-score of $-6.39 \mathrm{Kcal} / \mathrm{mol}$ ( Table 1), but with an EFE value equal to $-18.73 \mathrm{Kcal} / \mathrm{mol}$ higher than 11 ( Table 2), probably due to a consistent lack in ligand-target interactions for the absence of a glycoside portion ( Fig. 4; Fig. 1S, Supporting Information).

A superimposed model of the most stable docked poses of flavonoids $\mathbf{1 - 1 0}$ is reported in Fig. 2S, Supporting Information.

\section{Discussion}

\section{$\nabla$}

The DATATIPICA project was born with the purpose to use computational methods, such as VS, to identify new bioactive compounds early from an in-house ligand library, implemented in our laboratory, with the chemical entities typical of Bergamot and Tropea red onion Calabrian products. Our interest was not only in the flavonoids present in the nonvolatile fraction of these natural products, but the low-molecular weight compounds in the volatile fraction were also considered (Table 1S, Supporting Information).

The SBVS study was focused on a family of CA targets involved in several diseases, considering some human isoforms: ubiquitous hCA I and II, antiobesity target hCA VA, and cancer-related isoforms hCA IX and XII. The criteria for the selection of X-ray targets were: a) Homo sapiens as an organism, considering the next in vitro assays with human enzymes; b) the presence of a cocrystallized ligand (the most active when it was possible) not covalently bound to the target; c) a resolution $<2.50 \AA$; and d) the PDB depository year of the X-ray model.

The crystallographic model of mCA VA (PDB code 1DMY) does not respect the first requirement, because only two murine, truncated mitochondrial X-ray models are in the PDB. On the other hand, the comparison of the CA VA domain sequence between mice and humans shows a 78\% identity [29], so this model has been adopted as a structural model of the mCA VA isoform.

Against this isoform, we observed that glycoside polyphenols with a disaccharide portion $(\mathbf{3}, \mathbf{4}$, and $\mathbf{5})$ generally reached better EFE values, in the range of $-22.28 /-35.02 \mathrm{Kcal} / \mathrm{mol}$, than the monosaccharide derivative $8(-20.82 \mathrm{Kcal} / \mathrm{mol})$ and the non-glycosylated polyphenols (1, 2, 6, 7, 9, and 10), whose evaluations were around $-18.36 /-21.61 \mathrm{Kcal} / \mathrm{mol}$.

Eriocitrin (3), with the best EFE value, was identified in the in vitro assays as a new hCA VA inhibitor, and disaccharide derivatives 4 and 5 displayed better $K_{\mathrm{i}}$ values than other flavonoids, confirming that the disaccharide portion is involved in the inhibitory effect on hCA VA isoform ( Table 3). Apigenin (1) in vitro displays an inhibition performance not revealed by theoretical data, but among compounds $\mathbf{1 - 1 0}$, this compound showed the highest cLogP value (2.71), which is likely responsible for the unexpected biological result ( Table 3; Table 2S, Supporting Information). About the mechanism of action of hCAIs, recently, a work by Supuran's group reported four different types; but in regards to the flavonoids incorporating phenol moieties, they inhibit hCAs through a diverse, not yet determined, inhibition mechanism [23]. We hypothesized for this class of compounds the possibility to displace the water molecule coordinated to $\mathrm{ZN}$.

Therefore, when not considering all water molecules in the crystallographic model during docking simulations, it may be observed that the $\mathrm{C} 2$ catechol group of eriocitrin (3), not present in the other glycoside flavonoids $(\mathbf{4}, 5$ and $\mathbf{8})$, could directly coordi-

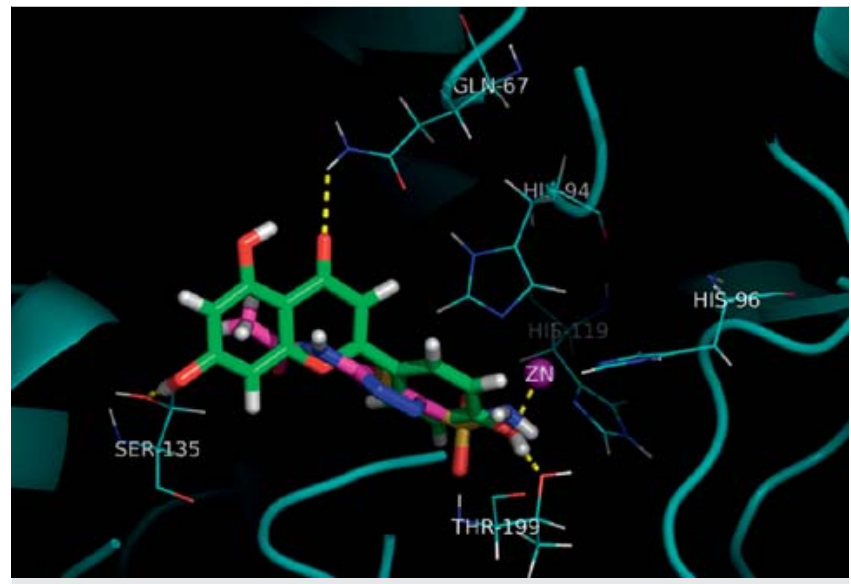

Fig. 4 Apigenin (1) best pose and its polar contacts/metal interaction into the mCA VA (PDB code 1DMY) binding cavity, superimposed with AZA (11). Apigenin (1) is rendered as carbon green sticks, AZA (11) as carbon pink sticks, the pocket amino acids are labeled as carbon cyan lines, the zinc atom as a purple sphere, and the rest of the target is described as a cyan cartoon. All non-carbon atoms are colored according to atom types. (Color figures available online only.)

nate the ZN of the mCA VA isoform, suggesting that displacement of a water molecule can occur in the recognition process (ه Fig. 3).

The best 3-mCA VA pose is mostly attributed to good contacts established mainly by the catechol and rutinoside portions. The former produces additional $\mathrm{H}$-bond interactions with the enzymatic residue THR199 located in the catalytic site. The latter is characterized by an extended H-bond network with several external mCA VA amino acid residues (GLN67, GLU69, GLN92, ASP171) at the entrance of the binding pocket. Details about the intermolecular H-bond distances are reported in Table 35, Supporting Information.

The bottom of the active site cavity is very much conserved in the 16 hCA isozymes, whereas the regions with the highest variation in amino acid sequence, consequently with the highest degree of structural diversity, are close to the entrance of the active site [23]. This is the reason of the nonspecific AZA inhibition toward the hCA isoforms. Conversely, it explains eriocitrin (3) selectivity, supported by the recognition properties of its glycoside portion interacting well with the hCA VA isozyme external residues (๑ Fig. 3).

The docking simulations carried out with apigenin (1) show that only its $\mathrm{C} 2$ phenolic group is involved in the zinc coordination into the mCA VA active site ( $\odot$ Fig. 4). The best pose stabilization is attributed, in this case, at the catalytic and entrance areas, respectively, to the intermolecular H-bonds with THR199, SER135, and GLN67 CA residues (Fig. 1S and Table 4S, Supporting Information).

In vitro results and hCA I, II, VA, IX, and XII inhibition by compounds 1-10 with respect to AZA (11) are reported in Table 3. In regards to the carbonic anhydrases (hCA VA and VB) exerting biological activities at the mitochondria level [30-32], only hCA VA was used, since no X-ray model of the other isoform, human or murine, is available.

The results of our study suggest that some Bergamot flavonoids may inhibit a mitochondrial hCA VA isoform with a competitive mechanism of action, coordinating the enzymatic ZN and establishing other non-covalent interactions, mainly via H-bonds. 
Eriocitrin (3) and apigenin (1), due to their inhibition profile against the hCA VA isoform, could be potential hit candidates for antiobesity treatment and prevention.

Pharmacological results reported for flavonoids such as apigenin (1), luteolin, and eriocitrin (3) already confirmed the beneficial effects against adipogenesis [33] and dyslipidemia, decreasing lipid droplets in the liver and inducing gene expression related to mitochondrial function in HepG2 cells [34]. These observations are in agreement with the mitochondrial involvement, and our study provides an enzymatic explanation of the molecular mechanism of action for some flavonoids.

In summary, in this work, theoretical and experimental approaches were integrated to identify natural bioactive compounds consistently present in some peculiar Calabrian cultivars, such as Bergamot and Tropea red onions, endowed with inhibition properties against the hCA VA target. In particular, the powerful potentiality of the virtual screening techniques seems to confirm its important role with the aim to speed up the identification of bioactive compounds useful for both nutraceutical and drug discovery purposes.

\section{Materials and Methods \\ $\nabla$}

\section{Chemicals}

All natural compounds and protein assay reagents were obtained from Sigma-Aldrich Co.

\section{Ligand library preparation}

Considering $C$. bergamia and $A$. cepa $\mathrm{L}$. var. Tropea as resources of natural compounds with potential therapeutic effects, for this work, a molecular database of their typical contents was generated using free access to the PUBCHEM [35] internet site. A 3D SDF file of 128 compounds (Table 1S, Supporting Information) was generated and imported into the graphic interface Maestro Schrödinger 9.7 [36]. The database was not only implemented with the glycoside compounds, but the aglycone structure for each of them was considered as well. In this way, the possible metabolites of the natural ligands after a hydrolytic reaction were also taken into account. All compounds were prepared as following: protonated forms of each molecule were calculated by means of the "LigPrep" [37] module, using additional protonated and tautomeric forms in the range of pH 5-9 with the default setting for the remaining parameters, ending up with a library of 280 compounds. Considering that for an in silico filtering experiment, a 3D structure database requires an efficient generation of reasonable, energetically minimized conformations [38], all ligands were also energy minimized in the absence of the receptor with the OPLS_2005 force field as implemented in the Schrödinger suite, and the lowest energy conformations were used as starting points for the docking simulations.

\section{Target: carbonic anhydrase isoform preparation}

An inevitable prerequisite for generating a structure-based model is the knowledge about the ligand-target interaction [39], including the availability of the 3D structure of the target. Docking experiments were performed using the X-ray crystallographic structures of five CA isoforms downloaded from the PDB [13]: hCA I, hCA II, mCA VA, hCA IX, and hCA XII with the respective PDB codes 1AZM [40], 4CQ0 [41], 1DMY [42], 3IAI [43], and 4HT2 [44].
For each X-ray model, the preparation procedure started with the "Protein Preparation Wizard" [45] function in Maestro 9.7 [36]. To study all interactions of each target with the ligands dataset, water molecules and nonprotein residues were removed. Subsequently, a refined procedure to fill in the missing side chains using the "Prime" module and the energy minimization of all hydrogen atoms completed the protocol. Hydrogen atoms were added and, finally, the energy minimization was performed using OPLS_2005 force field until the RMSD of all heavy atoms was within 0.3 Å of the X-ray PDB model.

\section{Docking simulation}

Docking studies of the natural library were performed with the software Glide 6.2 [46] on the CA crystal structures. The purpose of this method was to develop an exhaustive search in the space of the various positions, orientations, and conformations of each ligand compared to the targets.

A semiflexible approach was considered; all flexible 3D ligands from the chemical database were sequentially docked into the binding pocket of the rigid receptors. For each target, a cube of $35 \AA$ A length was defined as the binding region using the Receptor Grid Generation panel with the default options, centered on the former positions of the removed co-crystallized ligand with the protein into the catalytic site. Each docking run recorded a maximum of 10 poses per ligand. The Glide SP protocol was used, and the best docked poses for the novel hCAIs were selected, taking the most negative G-score value. In order to check the precision of Glide SP, re-docking simulations were performed, and we observed a good ability of this docking program to reproduce the binding modes of the original PDB entries, with very low RMSD values between the experimental X-ray structure and the best pose (1AZM PBD entry: 0.3751 $\AA$; 4CQ0 PDB entry: $1.3362 \AA$; 1DMY PBD entry: $0.3583 \AA$; 3 IAI PDB entry: $0.5019 \AA \AA$; 4 HT2 PDB entry: $0.7485 \AA$ ). All of the best docked poses of the dataset were subjected to the Embrace minimization utility, available in the Schrödinger Macromodel program [27], which was performed using the energy difference mode, with the specific water treatment. The Pymol 1.7.0.0 [28] molecular graphics system was used to visualize the molecules, the results of the docking experiments, and to generate all figures.

\section{In vitro hCA inhibition assay}

All hCA isoforms (I, II, VA, IX, and XII) used for the in vitro assays were purified and obtained in-house as previously reported in other works [30-32].

Hit compounds were tested in order to evaluate their biological activity against the hCA isoforms ( $\bigcirc$ Table 3). An Applied Photophysics stopped-flow instrument has been used for assaying the CA-catalyzed $\mathrm{CO}_{2}$ hydration activity [47]. Compounds 1-11 were tested in triplicate at each concentration used. Four inhibitor concentrations were used, starting from $10^{-5} \mathrm{M}$ to $10^{-8} \mathrm{M}$.

$\mathrm{IC}_{50}$ values were obtained from dose-response curves working at seven different concentrations of the test compound by fitting the curves using PRISM (www.graphpad.com) and nonlinear least squares methods; values represent the mean of at least three different determinations as previously described by us [48]. The inhibition constants $\left(K_{\mathrm{i}}\right)$ were then derived by using the Cheng-Prusoff equation [49] as follows:

$K_{\mathrm{i}}=\mathrm{IC}_{50} /\left(1+[\mathrm{S}] / \mathrm{K}_{\mathrm{m}}\right)$ 
where [S] represents the $\mathrm{CO}_{2}$ concentration at which the measurement was carried out, and $\mathrm{K}_{\mathrm{m}}$ is the concentration of substrate at which the enzyme activity is at half-maximal. The concentrations of enzymes used in the assay were as follows: hCA I: 13.2 nM, hCA II: 8.0 nM, hCA VA: 14.8 nM, hCA IX: 6.7 nM, and hCA XII: 10.9 nM.

\section{CLogP prediction}

The lipophilicity was calculated for compounds 1-11 accordingly to the ChemAxon software in the free http://www.chemicalize. org site.

\section{Supporting information}

Fig. 1S and 2S show, respectively, the best pose of 1 with the surface receptor and superimposed 1-10 flavonoids into the binding pocket of mCA VA; Table 1S lists the chemical contents of Bergamot and Tropea red onion used to create the ligand database; Table $2 \mathrm{~S}$ reports the cLogP of compounds 1-11; Table $3 \mathrm{~S}$ and $4 \mathrm{~S}$ show the geometrical details of the MCA VA best poses for eriocitrin (3) and apigenin (1), respectively. These are available as Supporting Information.

\section{Acknowledgements}

$\nabla$

This work was supported by the Interregional Research Center for Food Safety and Health at Magna Græcia University of Catanzaro (MIUR PON a3_00359).

\section{Conflict of Interest}

$\nabla$

The authors declare no conflict of interest.

\section{References}

1 Newman DJ, Cragg GM. Natural products as sources of new drugs over the 30 years from 1981 to 2010. J Nat Prod 2012; 75: 311-335

2 Langer T, Hoffmann RD. Virtual screening: an effective tool for lead structure discovery? Curr Pharm Des 2001; 7: 509-527

3 Artese A, Alcaro S, Moraca F, Reina R, Ventura M, Costantino G, Beccari $A R$, Ortuso F. State-of-the-art and dissemination of computational tools for drug-design purposes: a survey among Italian academics and industrial institutions. Future Med Chem 2013; 5: 907-927

4 Lavecchia A, Di Giovanni C. Virtual screening strategies in drug discovery: a critical review. Current Med Chem 2013; 20: 2839-2860

5 Alcaro S, Musetti C, Distinto S, Casatti M, Zagotto G, Artese A, Parrotta L, Moraca F, Costa G, Ortuso F, Maccioni E, Sissi C. Identification and characterization of new DNA G-quadruplex binders selected by a combination of ligand and structure based virtual screening approaches. J Med Chem 2013; 56: 843-855

6 Artese A, Costa G, Ortuso F, Parrotta L, Alcaro S. Identification of new natural DNA G-quadruplex binders selected by a structure-based virtual screening approach. Molecules 2013; 18: 12051-12070

7 Statti GA, Conforti F, Sacchetti G, Muzzoli M, Agrimonti C, Menichini F. Chemical and biological diversity of Bergamot (Citrus bergamia) in relation to environmental factors. Fitoterapia 2004; 75: 212-216

8 Furia E, Naccarato A, Sindona G, Stabile G, Tagarelli A. Multielement fingerprinting as a tool in origin authentication of PGI food products: Tropea red onion. J Agric Food Chem 2011; 59: 8450-8457

9 Gennaro L, Leonardi C, Esposito F, Salucci N, Maiani G, Quaglia G, Fogliano $V$. Flavonoid and carbohydrate contents in Tropea red onions: effects of homelike peeling and storage. J Agric Food Chem 2002; 50: 1904-1910

10 Dorant E, Van den Brandt PA, Goldbohm RA, Sturmans F. Consumption of onions and a reduced risk of stomach carcinoma. Gastroenterology 1996; 110: 12
11 Suh HJ, Lee JM, Cho JS, Kim YS, Chung SH. Radical scavenging compounds in onion skin. Food Res Int 1999; 32: 659

12 Pernice R, Borriello G, Ferracane R, Borrelli RC, Cennamo F, Ritieni A. Bergamot: A source of natural antioxidants for functionalized fruit juices. Food Chem 2009; 112: 545-550

13 Tripoli E, La Guardia M, Giammanco S, Di Majo D, Giammanco M. Citrus flavonoids: Molecular structure, biological activity and nutritional properties: A review. Food Chem 2007; 104: 466-479

14 Mollace V, Sacco I, Janda E, Malara C, Ventrice D, Colica C, Visalli V, Muscoli S, Ragusa S, Muscoli C, Rotiroti D, Romeo F. Hypolipemic and hypoglycaemic activity of bergamot polyphenols: from animal models to human studies. Fitoterapia 2007; 82: 309-316

15 Supuran CT. Carbonic anhydrases: novel therapeutic applications for inhibitors and activators. Nat Rev Drug Discov 2008; 7: 168-181

16 Supuran CT, Scozzafava A. Carbonic anhydrases as targets for medicinal chemistry. Bioorg Med Chem 2007; 15: 4336-4350

17 Pastorekova S, Parkkila S, Pastorek J, Supuran CT. Carbonic anhydrases: current state of the art, therapeutic applications and future prospects. J Enz Inhib Med Chem 2004; 19: 199-229

18 Supuran CT, Scozzafava A, Conway J. Carbonic anhydrases: catalytic mechanisms, distribution and physiological roles. In: Supuran CT, Scozzafava A, Conway J, editors. Carbonic anhydrase: its inhibitors and activators. Boca Raton: CRC Press; 2004: 1-24

19 Supuran CT. Carbonic anhydrases as drug targets-an overview. Curr Top Med Chem 2007; 7: 825-833

20 Aggarwal M, Kondeti B, McKenna R. Insights towards sulfonamide drug specificity in $\alpha$-carbonic anhydrases. Bioorg Med Chem 2013; 21: 1526-1533

21 Alterio V, Fiore AD, D'Ambrosio K, Supuran CT, Simone GD. Multiple binding modes of inhibitors to carbonic anhydrases: how to design specific drugs targeting 15 different isoforms? Chem Rev 2012; 112: 4421-4468

22 Gieling RG, Williams KJ. Carbonic anhydrase IX as a target for metastatic disease. Biorg Med Chem 2013; 21: 1470-1476

23 Ekinci D, Karagoz L, Ekinci D, Senturk M, Supuran CT. Carbonic anhydrase inhibitors: in vitro inhibition of $\alpha$ isoforms (hCA I, hCA II, bCA III, hCA IV) by flavonoids. J Enz Inhib Med Chem 2013; 28: 283-288

$24 \mathrm{Koz}$ Ö, Ekinci D, Perrone A, Piacente S, Alankuş-Çalışkan Ö, Bedir E, Supuran $C T$. Analysis of saponins and phenolic compounds as inhibitors of $\alpha$-carbonic anhydrase isoenzymes. J Enz Inhib Med Chem 2013; 28: 412-417

25 Berman HM, Westbrook J, Feng Z, Gilliland G, Bhat TN, Weissig H, Shindyalov IN, Bourne PE. The Protein Data Bank. Nucleic Acids Res 2000; 28: $235-242$

26 Ma DL, Chana DSH, Leung CH. Molecular docking for virtual screening of natural product databases. Chem Sci 2011; 2: 1656

27 Software: MacroModel, version 10.3. New York: Schrödinger, LLC; 2014

28 Software: PyMOL Molecular Graphics System, Version 1.7.0.0. New York: Schrödinger, LLC; 2014

29 Shah GN, Hewett-Emmett D, Grubb JH, Migas MC, Fleming RE, Waheed A, Sly WS. Mitochondrial carbonic anhydrase CA VB: differences in tissue distribution and pattern of evolution from those of CA VA suggest distinct physiological roles. PNAS 2000; 97: 1677-1682

30 De Simone G, Supuran CT. Antiobesity carbonic anhydrase inhibitors. Curr Top Med Chem 2007; 7: 879-884

31 Poulsen SA, Wilkinson BL, Innocenti A, Vullo D, Supuran CT. Inhibition of human mitochondrial carbonic anhydrases VA and VB with para-(4phenyltriazole-1-yl)-benzenesulfonamide derivatives. Bioorg Med Chem Lett 2008; 18: 4624-4627

32 D'Ascenzio M, Carradori S, De Monte C, Secci D, Ceruso M, Supuran CT. Design, synthesis and evaluation of $\mathrm{N}$-substituted saccharin derivatives as selective inhibitors of tumor-associated carbonic anhydrase XII. Bioorg Med Chem 2014; 22: 1821-1831

33 Kim JP, Lee I, Seo J, Jung M, Kim Y, Yim N, Bae K. Vitexin, orientin and other flavonoids from Spirodela polyrhiza inhibit adipogenesis in 3T3L1 cells. Phytother Res 2010; 24: 1543-1548

34 Hiramitsu M, Shimada Y, Kuroyanagi J, Inoue T, Katagiri T, Zang L, Nishimura Y, Nishimura N, Tanaka T. Eriocitrin ameliorates diet-induced hepatic steatosis with activation of mitochondrial biogenesis. Sci Rep 2014; 4: 3708

35 PUBCHEM free web site. Available at http://pubchem.ncbi.nlm.nih.gov. Acessed January 10, 2014

36 Software: Maestro, version 9.7. New York: Schrödinger, LLC; 2014

37 Software: LigPrep, version 2.9. New York: Schrödinger, LLC; 2014 
38 Kirchmair J, Laggner C, Wolber G, Langer T. Comparative analysis of protein-bound ligand conformations with respect to catalyst's conformational space subsampling algorithms. J Chem Inf Model 2005; 45: 422430

39 Congreve M, Murray CW, Blundell TL. Structural biology and drug discovery. Drug Disc Today 2005; 10: 895-907

40 Chakravarty S, Kannan KK. Drug-protein interactions. Refined structures of three sulfonamide drug complexes of human carbonic anhydrase I enzyme. J Mol Biol 1994; 243: 298-309

41 Moeker J, Peat TS, Bornaghi LF, Vullo D, Supuran CT, Poulsen SA. Cyclic secondary sulfonamides: unusually good inhibitors of cancer-related carbonic anhydrase enzymes. J Med Chem 2014; 57: 3522-3531

42 Boriack-Sjodin PA, Heck RW, Laipis PJ, Silverman DN, Christianson DW. Structure determination of murine mitochondrial carbonic anhydrase V at 2.45-A resolution: implications for catalytic proton transfer and inhibitor design. Proc Natl Acad Sci U S A 1995; 92: 10949-10953

43 Alterio V, Hilvo M, Di Fiore A, Supuran CT. Crystal structure of the catalytic domain of the tumor-associated human carbonic anhydrase IX. Proc Natl Acad Sci U S A 2009; 106: 16233-16238
44 Dudutiené V, Zubrienė A, Smirnov A, Gylytė J, Timm D, Manakova E, Gražulis S, Matulis D. 4-Substituted-2,3,5,6-tetrafluorobenzenesulfonamides as inhibitors of carbonic anhydrases I, II, VII, XII, and XIII. Bioorg Med Chem 2013; 21: 2093-2106

45 Software: Schrödinger Suite 2013 Protein Preparation Wizard; Epik version 2.7. New York: Schrödinger, LLC; 2013; Impact version 6.2. New York: Schrödinger, LLC; 2013; Prime version 3.4. New York: Schrödinger, LLC; 2014

46 Software: Glide, version 6.2. New York: Schrödinger, LLC; 2014

47 Khalifah RG. The carbon dioxide hydration activity of carbonic anhydrase. I. Stop-flow kinetic studies on the native human isoenzymes B and C. J Biol Chem 1971; 246: 2561-2573

48 Lopez M, Drillaud N, Bornaghi LF, Poulsen SA. Synthesis of S-glycosyl primary sulfonamides. J Org Chem 2009; 74: 2811-2816

49 Cheng $H C$. The power issue: determination of $\mathrm{KB}$ or Ki from IC $\mathrm{C}_{50}$. A closer look at the Cheng-Prusoff equation, the Schild plot and related power equations. J Pharmacol Toxicol Methods 2001; 46: 61-71 